\title{
The Impact of Noise Label on Beampattern and SINR of MVDR Beamformer
}

\author{
Suhail Najm Shahab ${ }^{1}$, Ayib Rosdi Zainun ${ }^{1}$, Izzeldin Ibrahim Mohamed ${ }^{1}$, and Nurul Hazlina Noordin ${ }^{1}$
}

\begin{abstract}
Minimum Variance Distortionless Response (MVDR) is basically a unity gain adaptive beamformer which is suffered from performance degradation due to the presence of interference and noise. Also, MVDR is sensitive to errors such as the steering vector errors, and the nulling level. MVDR combined with a linear antenna array (LAA) is used to acquire desired signals and suppress the interference and noise. This paper examines the impact of the noise variance label $(\sigma n 2)$ and the number of interference sources by using Signal to Interference plus Noise Ratio (SINR) and array beampattern as two different Figure-of-Merits to measure the performance of the MVDR beamformer with a fixed number of array elements (L). The findings of this study indicate that the MVDR have successfully placed nulls in the nonlook angle with average SINR of 99.6, 49.6, $24.9 \mathrm{~dB} d B$ for on2.of $-50,0,50$ $\mathrm{dB}$, respectively. Also, the MVDR provides accurate majorlobe to the real user direction, even the $\sigma \mathrm{n} 2$ are bigger than desired signal power. The proposed method was found to perform better than some existing techniques. Based on this analysis, the beampattern is not heavily relies on the on 2 . Moreover, the SINR strongly depends on the $\sigma \mathrm{n} 2$ and the number of SNOIs.
\end{abstract}

Keywords-Beamforming, Linear antenna array, Minimum variance distortionless response, SINR, Smart antenna.

\section{INTRODUCTION}

$\mathrm{C}_{\mathrm{o}}^{\mathrm{c}}$ URRENTLY, the mobile cellular networks are experiencing a massive evolution of data traffic, because of multi-media and internet applications that are used by a vast number of devices such as smartphones, mobile PC and tablets [1], [2]. Most beamforming techniques have been considered for use at the base station (BS) since anten-na arrays are not feasible at mobile terminals due to space limitations [3]. The Long Term Evolution (LTE), as introduced by the 3rd Generation Partnership Project (3GPP), is an extremely flexible radio interface, the first LTE deployment was in 2011. LTE is the evolution of 3GPP Universal Mobile Telecommunication System (UMTS) towards an all-IP network to ensure the competitiveness of UMTS for the next ten years and beyond. LTE was developed in Release 8 and 9 of the 3GPP specification. Maravedis, anticipates that 3 LTE-TDD and 59 LTE-FDD networks will be running worldwide by the end of 2011. By 2016, there will be 305 million LTE subscribers, which means about 44 million (14\%) will be LTE-TDD clients and the remaining 261 million (86\%) will be LTE-FDD [4].

${ }^{1}$ Faculty of Electrical and Electronics Engineering, Universiti Malaysia Pahang, 26600, Pahang, Malaysia.
Interference is one of the significant obstacles in the wireless networks. It can be caused by other users or by the signal itself [5]. The signal can interfere with itself due to multipath components, where the signal is gathere-d with another version of the signal that is delayed because of another propagation path [6]. The fundamental pr-inciple of the Adaptive beamforming ( $\mathrm{ABF}$ ) algorithm is to track the statistics of the surrounding interference and noise field as well as adaptively placing nulls that decrease dramatically the interference and noise under the restriction that the look angle is not distorted at the beamformer's output [7]. The basic idea of the Minimum Va-riance Distortionless Response (MVDR) algorithm is to estimate the beamforming excitation coefficients in an adaptive way by minimizing the variance of the residual interference and noise whilst enforcing a set of linear constraints to ensure that the real user signal are not distorted [7].

The authors in [8] proposed an enhanced model of MVDR algorithm by replace the position of the reference element in steering vector to be in the central of the array and the number of elements must be odd. Simulation results show that modified MVDR has a realistic behavior especially for detecting the incoming signals direction and outperforms the conventional MVDR. One of the popular approaches to improve the classic Capon beamfor-mer in the presence of finite sample effect and steering vector errors is the diagonal loading, which was studied by [9]. The idea behind diagonal loading is to adapt a covariance matrix by adding a displacement value to the diagonal elements of the estimated covariance matrix. Nevertheless, how to select an appropriate diagonal loadi-ng level is a challenging task. [10] mentioned that the element spacing must be $d \leq \lambda / 2$ to prevent spatial aliasin-g. In [11], the author presents a comparative study of MVDR algorithm and LMS algorithm, where results show that LMS is the better performer. The SINR maximization is another criterion employed in the joint transmitter and receiver beamforming algorithms [12], [13], [14]. In an analysis of [15], the mixing of differential algorithm based LAA is applied to deepen nulls and lower side lobe levels (SLLs) in the unwanted direction, and they fou-nd the max null depth of $-63 \mathrm{~dB}$ by using 20 element. The statistic numerical algorithm was proposed to obtain the requirement for the amplitude and phase error of multibeam active phased array antenna [16]. The radiation beampattern is simulated from the value of the random amplitude and phase errors of phase shifter. From the results, it is found that the only way to meet the requirement of the sidelobe level is to use digital 
beamforming. The researchers in [7] investigate the performance of the MVDR beamformer for four different types of noise and source incidence angles using SNR and beampattern as the evaluation criteria. An evaluation of the trade-off between noise reduction and reverberation of the MVDR filter is presented in [17].

The DOA estimation is used to specify the main directions of all incoming signals. A previous research has an-alyzed the accuracy and precision of a proposed wideband capon beamforming for estimating the elevation, azi-muth angles and velocity for target parameters using planner antenna array [18]. The function of $\mathrm{ABF}$ algorithm is to form the mainbeam to the user direction and placing nulls towards interference and noise directions by adj-usting the antenna itself using beamforming (BF) techniques to achieve better transmission or reception beampa-ttern which increases SINR by mitigating co-channel interference present in the wireless communication system.

So far, this work considers problem dealing with the impact of the noise label, number of undesired signals and the angular separation between real desired user and undesired signals. It is important to investigate the impact of SNOIs on the beampattern that can offer the best BF capabilities in terms of directing the majorlobe toward the Signal of Interest (SOI) direction while nulls towards the Signal Not of Interest (SNOI) direction. In this paper, for a fixed number of antenna elements, in order to evaluate the performance of the MVDR algorithm applied to the ABF problem of LAA, the scenarios proposed are mainly presented in terms of extremely adverse conditions, i.e. by increasing the noise label to exceed the desired signal power over which the array antenna whilst precisely steering the majorbeam toward the specified direction with an isolation from the null, and by rejecting interference presented on the array system. Whereas no complete assessment of the SINR and the beampattern as a function of all the above mentioned parameters. The analysis of the MVDR in this work is carried out in two different scenarios where the MVDR performance is assessed with two important metrics; SINR and beampattern. The remainder of this paper is organized as follows. In section 2, MVDR beamformer based on linear antenna array design method with the signal propagation model is described. The simulation results and performance analysis are provided in Section 3. Finally, in Section 4, the paper's conclusions and summary of MVDR performance are described.

\section{SYSTEM MODEL \& MVDR BEAMFORMING}

In this section, the mathematical formulation of the design model for adaptive beamforming will be presented in detail. Consider a single cell with $L$ elements antenna array. Let there be $S$ wanted signal sources and $I$ interference sources spreading on same the frequency channel at the same time. The algorithm starts by creating a real life signal model. A number of plane waves are considered from $K$ narrowband sources impinging from various angles $(\theta, \phi)$. The impinging radio frequency signal reaches into antenna array from far field to the array geometry of linear antenna arrays (LAAs).
After the signals are received by antenna arrays consisting of the wanted user signal, the interference source, and the noise, the first part is to estimate the direction of the arrival of the $S$ signal and $I$ signals using a well-known algorithm developed by capon, named MVDR spectrum estimator, to find the DOA angles of several sources. However, the MVDR estimator algorithm wants information of the number of sources. With the known direction of the source, then the second part is applied by using MVDR ABF technique that places a straight beam to $S$ signal and placing nulls in the direction of $I$ signals. Each signal is multiplied by adaptable complex weights and then summed to form the system output.

The total composite signals received by an adaptive antenna array at time index, $t$, become:

$$
\begin{gathered}
x_{T}(t)=\sum_{s=1}^{S} x_{s}(t)+\sum_{i=1}^{I} x_{i}(t)+x_{n} \\
=\sum_{s=1}^{S} x_{s}(t) a\left(\theta_{s}\right)+\sum_{i=1}^{I} x_{i}(t) a\left(\theta_{i}\right)+x_{n}
\end{gathered}
$$

Where $x_{\mathrm{T}}(t) \in C^{K \times L}, x_{s}(t), x_{i}(t), x_{n}(t)$, denote the desired signal, interference signal and noise added from White Gaussian noise, respectively. The unwanted signal consists of $x_{i}(t)+x_{n}(t)$ and $I$ is the number of interferences, the desired angle and interference direction of arrival angles are $\theta_{s}$ and $\theta_{i}$ , $i=1,2 \ldots, I$, respectively. a $\left(\theta_{s}\right)$ denote the steering vector or array response for wanted signal while $\mathrm{a}\left(\theta_{i}\right)$ refers to the interference signal steering vector or array response to the unwanted signal. Steering vector is a complex vector $\in C^{L \times K}$ containing the responses of all elements of the array to a narrowband source of unit power depending on the incident angle, which is given by [19]:

$$
a(\theta)=\left[1, e^{-j q d \sin (\theta)}, e^{-j q 2 d \sin (\theta)}, \ldots, e^{-j q(L-1) d \sin (\theta)}\right]
$$

Where $\mathrm{j}$ is the imaginary unit, (i.e. $\mathrm{j}^{2}=-1$ ), $d$ is the spacing between elements and $q$ is the wave number given as $2 \pi / \lambda$. Where $\lambda$ refers to the received signal wavelength. The signal $x_{T}(t)$ received by multiple antenna elements is multiplied with a series of amplitude and phase (weight vector coefficients) which accordingly adjust the amplitude and phase of the incoming signal. This weighted signal is a linear combination of the data at $L$ elements, resulting in the array output, $y(t)$ at any time $t$, of a narrowband beamformer, which is given by;

$$
y(t)=\sum_{l=1}^{L} w^{H} x_{T}(t)
$$

Where $y(t)$ is the beamformer output, $x_{T}(t)$ is the antenna element's output, $\mathrm{w}$ is the complex weight vector for the antenna element $=\left[w_{1}, w_{2}, \ldots, w_{L}\right]^{T}$ is $\in C^{L \times 1}$ beamforming complex vector. $(.)^{\mathrm{H}}$ and $(.)^{\mathrm{T}}$ denotes the conjugate transpose (Hermitian transpose) of a vector or a matrix, which is used to simplify the mathematical notation and transposes operators respectively. The weight vector at time $t+1$ for any system that uses the immediate gradient vector $\nabla \xi(t)$ for weight vector upgrading and evades the matrix inverse operation, which is defined as follows:

$$
w(t+1)=w(t)+(1 / 2) \mu[\nabla \xi(t)]
$$

Where $\mu$ is the step size parameter, the convergence speed control by $\mu$ and lies between 0 and 1 . The smallest values of $\mu$ facilitate the high-quality estimation and sluggish 
concurrence, while huge values may result in a rapid union. However, the constancy over the minimum value may disappear, consider $0<\mu<1 / \lambda$. An instantaneous estimation of gradient vector is written as

$$
\begin{gathered}
\nabla \xi(t)=-2 p(t)+2 R_{y}(t) W(t) \\
p(t)=d^{*}(t) x_{T}(t)
\end{gathered}
$$

A precise calculation of $\nabla \xi(\mathrm{t})$ is not possible because prior information on cross-correlation vector, $p$ and covariance matrix, $R_{\mathrm{y}}$ of the measurement vector are required. By substituting (8) with (6), the weight vector is derived as follows:

$$
\begin{aligned}
W(t+1) & =W(t)+\mu[p(t)-R(t) W(t)] \\
= & W(t)+\mu x_{T}(t)\left[d^{*}(t)-x_{T}(t) W(t)\right] \\
= & W(t)+\mu x_{T} e^{*}(t)
\end{aligned}
$$

The following formulas can further define the desired signal:

$$
\begin{gathered}
y(t)=w^{H}(t) x_{T}(t) \\
e(t)=d(t) \cdot y(t) W(t+1) \\
=W(t)+\mu x_{T}(t) e^{*}(t)
\end{gathered}
$$

The covariance matrix, $R_{y}$ is constructed conventionally with unlimited snapshots. However, it is estimated by using the limited snapshots signal in the actual application. It can be expressed as:

$$
\begin{gathered}
R_{y}=\sum_{s=1}^{S} \sigma_{s}^{2} a\left(\theta_{s}\right) a^{H}\left(\theta_{s}\right)+\sum_{i=1}^{I} \sigma_{i}^{2} a\left(\theta_{i}\right) a^{H}\left(\theta_{i}\right) \\
R_{s}=\sum_{s=1}^{S} \sigma_{s}^{2} a\left(\theta_{s}\right) a^{H}\left(\theta_{s}\right) \\
R_{i+n}=\sum_{i=1}^{I} \sigma_{i}^{2} a\left(\theta_{i}\right) a^{H}\left(\theta_{i}\right)+\sigma_{n}^{2} I d_{L} \\
R_{y}=R_{s}+R_{i+n}=\mathrm{E}\left[x_{T}(t) x_{T}{ }^{H}(t)\right] \\
\sigma_{s}^{2}=E\left[\left|x_{s}(t)\right|^{2}\right], \sigma_{i}^{2}=E\left[\left|x_{i}(t)\right|^{2}\right]
\end{gathered}
$$

where $R_{y}, \sigma_{s}^{2}, \sigma_{i}^{2}, \sigma_{n}^{2}, I d_{L}, R_{s}, R_{i+n}$ and $E[$.$] denotes,$ respectively, the $L \times L$ theoretical covariance matrix, power of the desired signal, interference power, noise power, $L \times L$ identity matrix, SOI covariance matrix, interference plus noise covariance matrix and expectation operator. The most common formulation of the MVDR beamformer that determines the $L \times 1$ optimum weight vector is the solution to the following constrained problem [20]:

$$
\begin{gathered}
W_{M V D R}=\arg _{W_{M V D R}} \min \left(W^{H} R_{y} W\right)=\min \mathrm{E}\left[|y(t)|^{2}\right] \\
\Rightarrow \min _{w} P(\theta)=\left\{w^{H} R_{y} w\right\} \quad \text { s.t. } \quad w^{H} a\left(\theta_{s}\right)=1
\end{gathered}
$$

Where $P(\theta)$ denotes the mean output power, a beampattern can be given in $\mathrm{dB}$ as [21]:

$$
\text { beampattern }=20 \log _{10}(|P(\theta)| / \max |P(\theta)|)
$$

This method reduces the contribution of the unwanted signal by minimizing the power of output undesired and ensuring the power of useful signal equals to 1 (constant) in the direction of useful signal $w^{H} a\left(\theta_{s}\right)=1$. By using Lagrange multiplier, the MVDR weight vector that gives the solution for the above equation as per the following formula [22]:

$$
w_{M V D R}=R_{y}^{-1} a\left(\theta_{s}\right) / a^{H}\left(\theta_{s}\right) R_{y}^{-1} a\left(\theta_{s}\right)
$$

Inserting (22) into (12), the output of MVDR is given by;

$$
y(t)=w^{H}(t) x_{T}(t)=x_{s}(t)+w^{H} x_{i}(t) a\left(\theta_{i}\right)+w^{H} x_{n}(t)
$$

The output signal power of the array as a function of the DOA estimation, using optimum weight vector from MVDR beamforming method [23], it is given by MVDR spatial spectrum for angle of arrival estimated by detecting the peaks in this angular spectrum as [24]:

$$
P_{M V D R}(\theta)=1 / a^{H}\left(\theta_{s}\right) R_{y}^{-1} a\left(\theta_{s}\right)
$$

Finally, the SINR is defined as the ratio of the desired signal power divided by the undesired signal power:

$$
\operatorname{SINR}=\mathrm{E}\left\{\left|y_{s}(t)\right|^{2}\right\} / \mathrm{E}\left\{\left|y_{i+n}(t)\right|^{2}\right\}=w^{H} R_{s} w / w^{H} R_{i+n} w
$$

\section{Simulation Results AND ANALYSIS}

In this study, the performance of the proposed MVDR beamformer technique is presented, and illustrative of the array of L linear antenna element configuration is arranged along some axis added to the beamformer system at the cellular base station (BS). The array receives signals from different spatially separated users. The received signal $d_{\text {qonsists of the intended signal, co-channel interference, and a }}$ random noise component. To increase the desired signal output power and reduce the power of the unwanted signal, $\mathrm{BF}$ is employed at the BS. The antenna array elements separated by interelement spacing, $\mathrm{d}$, at carrier frequency $(\mathrm{Fc})$ of $2.6 \mathrm{GHz}$, which is the spectrum band allocated to LTE operators in Malaysia [25]. To measure the performance of the MVDR algorithm for ABF applications with varying noise variance label, null deepth and the accuracy to suppress the interference source. The goal is to find out the effects of noise label and analyzed on intefereing signals to achieve the best beamforming capabilities that form the majorbeam in the wanted direction and null in the directions of interference with highest output SINR. The incident fields are assumed to impinge from different angle of arrival (AoA) $\theta$ s and $\theta i=\{1$, $2, \ldots, \mathrm{L}-1\}$ in the azimuth plane $(-\pi / 2<\theta<\pi / 2)$. Throughout the simulations, the array is illuminated by uncorrelated sources of equally power levels. Three different scenarios are considered, and the simulation parameters setting in this study is shown in Table 1. 
TABLE 1

KEY SIMULATION PARAMETERS OF MVDR BEAMFORMER

\begin{tabular}{lclc}
\hline \hline $\begin{array}{l}\text { Key system } \\
\text { parameters }\end{array}$ & Values & Key system parameters & $\begin{array}{c}\text { Value } \\
\mathrm{s}\end{array}$ \\
\hline $\begin{array}{l}\text { Array antenna } \\
\text { configuration }\end{array}$ & LAA & \# SOI & 1 \\
Antenna type & Isotropic & \# SNOIs & 1,2, \\
$\begin{array}{l}\text { Carrier frequency } \\
\text { (Fc) }\end{array}$ & $2.6 \mathrm{GHz}$ & SIR [dB] & 3 \\
$\begin{array}{l}\text { Beam scanning } \\
\text { range }\end{array}$ & $\begin{array}{c} \pm 90^{\circ} \\
\begin{array}{l}\text { Numimuth) } \\
\text { (L) }\end{array}\end{array}$ & Snapshots (ns) & 0 \\
Element spacing (d) & $\lambda$ & $\begin{array}{l}\text { Noise power label } \sigma_{n} \\
{[\mathrm{~dB}]}\end{array}$ & $-50:$ \\
\hline \hline
\end{tabular}

\section{A. The First Scenario}

Using multiple antennas at the BS can reduce the effects of co-channel interference, multipath fading, and background noise. Many BF algorithms have been devised to cancel interference sources that appear in the cellular system. MVDR algorithm can place null at the interferences without any distortion to the real user path.

The first simulation scenario reveals the results calculated by considering the distance between array elements set to be $d=0.5 \lambda$ with Signal to Noise Ratio (SNR) equal to Interference to Noise Ratio (INR) $=-50: 50 \mathrm{~dB}$ and number of data samples $=250$. Figures $2(a, b, c)$ illustrate a typical $2 \mathrm{D}$ MVDR beampattern plot displayed in a rectangular coordinates, Uniform LAA with $L=8$ elements. Assume that the noise at each element is additive white, independent, complex zero mean and Gaussian with $\sigma_{n}{ }^{2}$. The SOI is considered to be a plane wave from the presumed direction $\theta_{s}$ of one desired signal with two interfering sources are assumed to have DOAs $\left(\theta_{i}\right)$ and the reference element at the one-end side of the array. Figure 1(a) shows one desired signal arriving from $\theta_{s}=-30^{\circ}$ and two interference arriving from $\theta_{i}=0^{\circ}$ and $30^{\circ}$. Figure $1(\mathrm{~b})$ displays one desired signal arriving from $\theta_{s}=0^{\circ}$ and two interference arriving from $\theta_{i}=$ $\pm 30^{\circ}$. Figure $1(\mathrm{c})$ demonstrations one desired signal arriving from $\theta_{s}=30^{\circ}$ and two interference arriving from $\theta_{i}=-30^{\circ}$ and $0^{\circ}$. Each received signal also includes noise. It can be seen clearly from these three plots is the that MVDR is robust against ${\sigma_{n}}^{2}$, as the noise power label goes higher the nulling level to the interference source become worse. Furthermore, MVDR successfully placed nulls at each of the interference sources and provides majorlobe formed to reach the real user direction accurately. The maximum depth null (Null) at interference direction and output SINR are shown in Table 2. The output SINR decreases as the $\sigma_{n}{ }^{2}$ increases as depicted in figure 1(d) for a range of ${\sigma_{n}}^{2}$ from -50 till $50 \mathrm{~dB}$. However, the reduction at higher values of $\sigma_{n}^{2}$ is much less at lower values of ${\sigma_{n}}^{2}$. The average SINR output for $\sigma_{n}{ }^{2}=-50,0$, and $50 \mathrm{~dB}$ founded to be $99.6,49.6$ and $24.9 \mathrm{~dB}$ while maximum null are $-105,-55$ and $-33.4 \mathrm{~dB}$, respectively as depicted in Table 2.

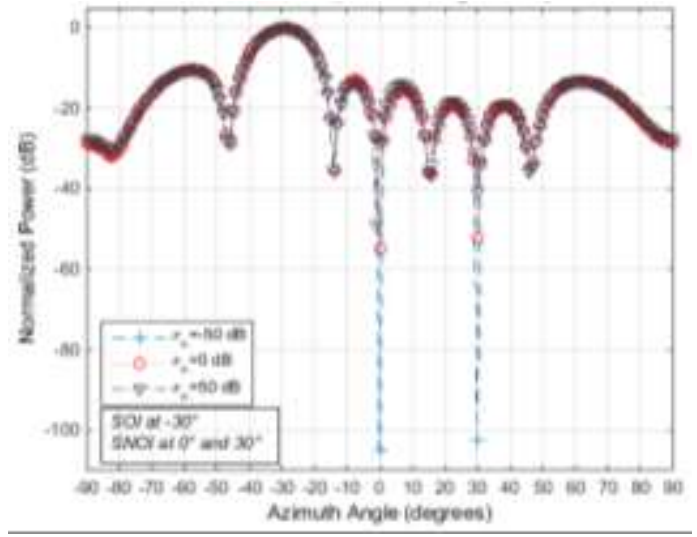

(a)

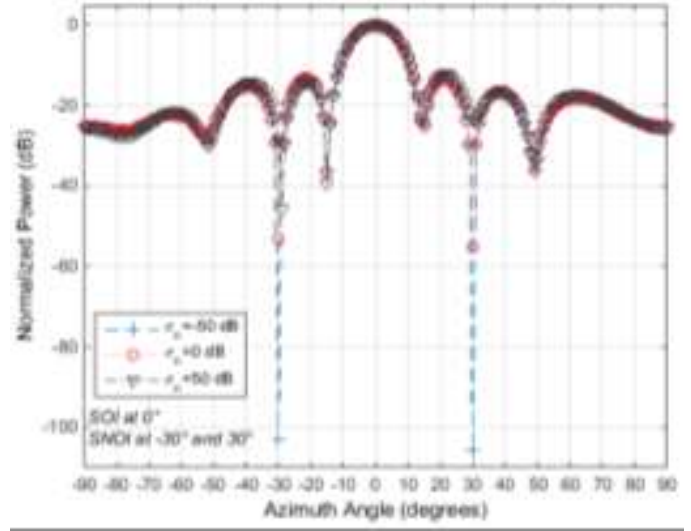

(b)

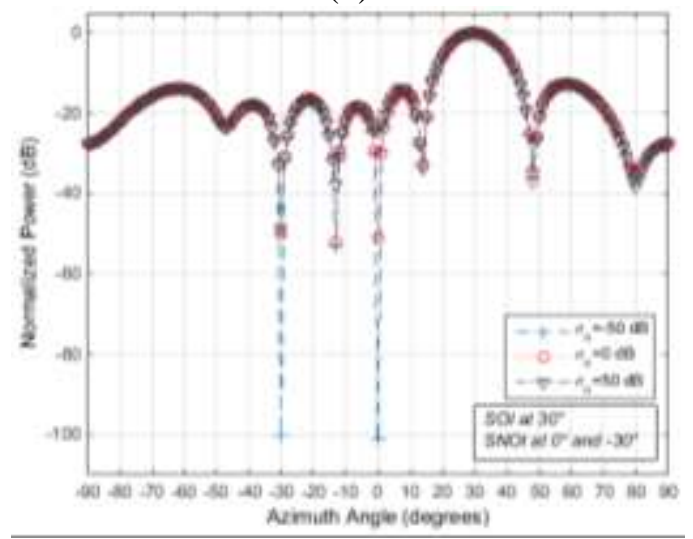

(c)

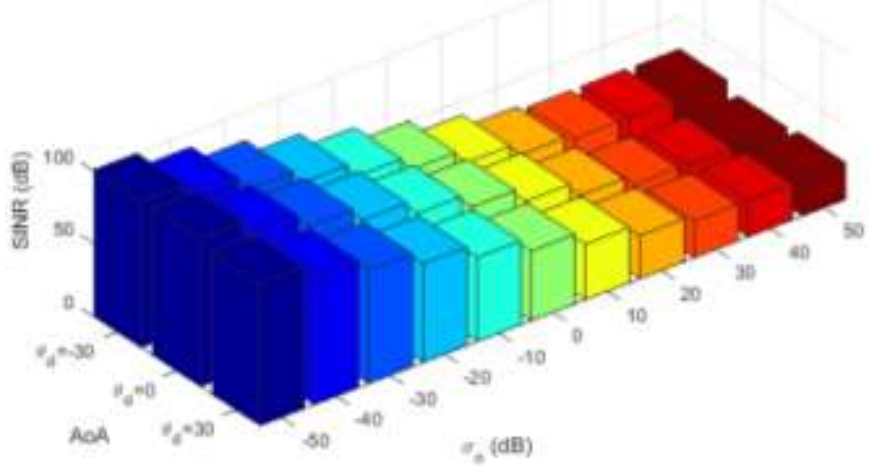

(d)

Fig. 1: Performance comparison of MVDR beampattern analysis of MVDR BF with $\mathrm{L}=8, \mathrm{~d}=\lambda / 2$ and $\mathrm{SNOIs}=2$ 
TABLE II

MVDR PERFORMANCE ANALYSIS FOR $\mathrm{L}=8, \mathrm{D}=\Lambda / 2$ AND $\Sigma_{N}^{2}=-50,0,50 \mathrm{DB}$.

\begin{tabular}{|c|c|c|c|c|c|c|c|c|c|c|c|c|c|c|c|c|}
\hline$\overline{\overline{\sigma_{n}{ }^{2}}}$ & $\theta_{s}$ & $\theta_{i}$ & Null & SINR & - & $\bar{~} \overline{\sigma_{n}{ }^{2}}$ & $\theta_{s}$ & $\theta_{i}$ & Null & SINR & - & $\bar{~} \overline{\sigma_{n}{ }^{2}}$ & $\theta_{s}$ & $\theta_{i}$ & Null & SINR \\
\hline-50 & \multirow{3}{*}{$-30^{\circ}$} & \multirow{3}{*}{$\begin{array}{c}0^{\circ} \\
30^{\circ}\end{array}$} & -104 & 100.2 & - & -50 & \multirow{3}{*}{$0^{\circ}$} & \multirow{3}{*}{ $\pm 30^{\circ}$} & -105 & 101.2 & - & -50 & \multirow{3}{*}{$30^{\circ}$} & \multirow{3}{*}{$\begin{array}{c}-30^{\circ} \\
0^{\circ}\end{array}$} & -101 & 97.64 \\
\hline 0 & & & -54 & 50.2 & - & 0 & & & -55 & 51.0 & - & 0 & & & -52 & 47.71 \\
\hline 50 & & & -40 & 27.9 & - & 50 & & & -28 & 22.4 & - & 50 & & & -49 & 24.67 \\
\hline
\end{tabular}

\section{B. The Second Scenario}

The subsequent MVDR pattern plots with cancelation for all interferences are shown in Figures 3(a, b, c). It sho-ws the beampattern for an 8-element linear array in the presence of a different angle of arrival (AoA) for SOI and SNOIs. The second simulation scenario demonstrates the MVDR behavior when the number of SNOIs incr-eases. Whereas, the performances of the MVDR method are examined in the case of SNOIs increases with noise. It is assumed that one desired source impinge on the array of the directions for an SOI at $\theta \mathrm{s}$ and different number of SNOIs ( $\theta$ i). Figure 2(a) shows one desired signal arriving from $\theta \mathrm{s}=-20^{\circ}$ and one interference arriving from $\theta \mathrm{i}=0^{\circ}$. Figure 2(b) displays one desired signal arriving from $\theta \mathrm{s}=0^{\circ}$ and two interference arriving from $\theta \mathrm{i}=-$ $15^{\circ}$ and $20^{\circ}$. Figure 2(c) demonstrations one desired signal arriving from $\theta \mathrm{s}=30^{\circ}$ and three interference arriving from $\theta \mathrm{i}=$ $-30^{\circ}, 0^{\circ}$ and $50^{\circ}$ in the azimuth plane and each received signal also includes noise. All other parameters are chose-n the same in the previous scenario. It is found that the majorlobe still directed to the real user direction with po-wer of $0 \mathrm{~dB}$ and the negative power strongly goes up as on 2 increases while the nulling level and SINR are stron-gly affected by SNOIs increases. In other words, sharper and deeper nulls would be produced and hence improve the SINR by adding more elements to the array. In addition, Table 3 compares the power values of SINR for these three cases. Moreover, increasing the number of elements results in narrower majorlobe beamwidth that is very useful in directing the antenna beam to the desired user while the number of nulls in the pattern increases. On the other hand, the computing operations become more complex. Besides, as the number of array sensors increases, the mean cost of design increases due to the increasing number of RF modules, A/D converters, and other components. This causes the operational power consumption to increase as well.

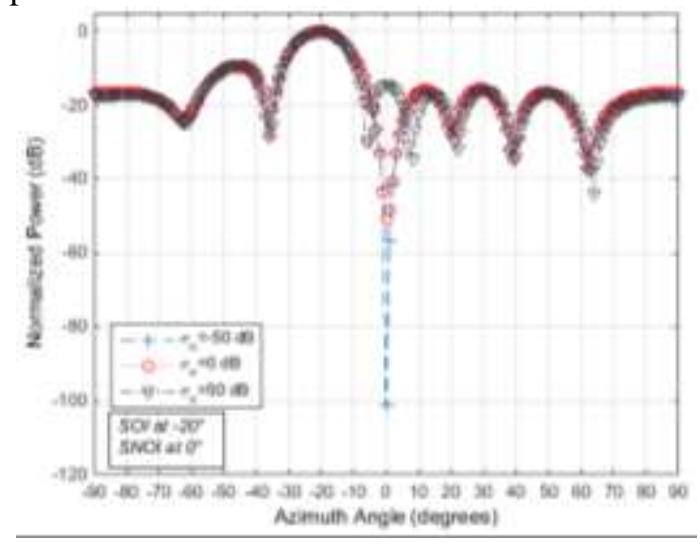

(a)

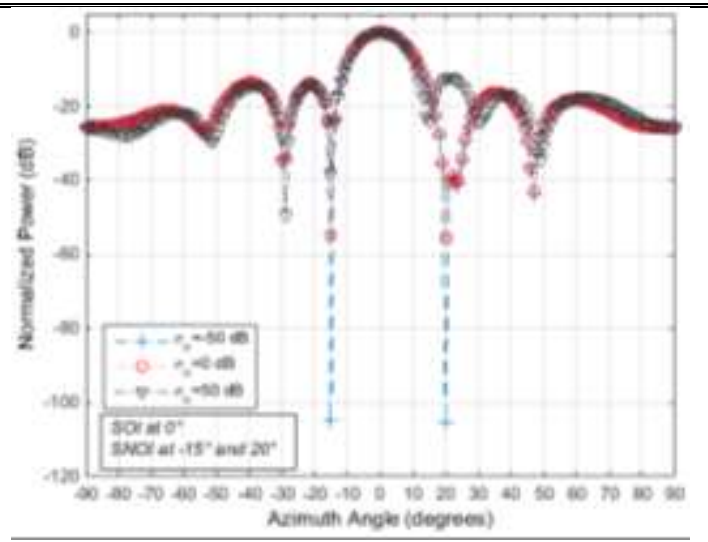

(b)

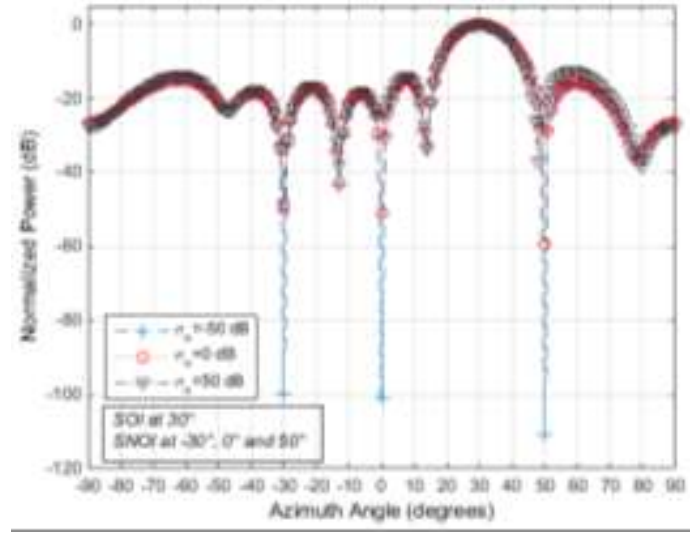

(c)

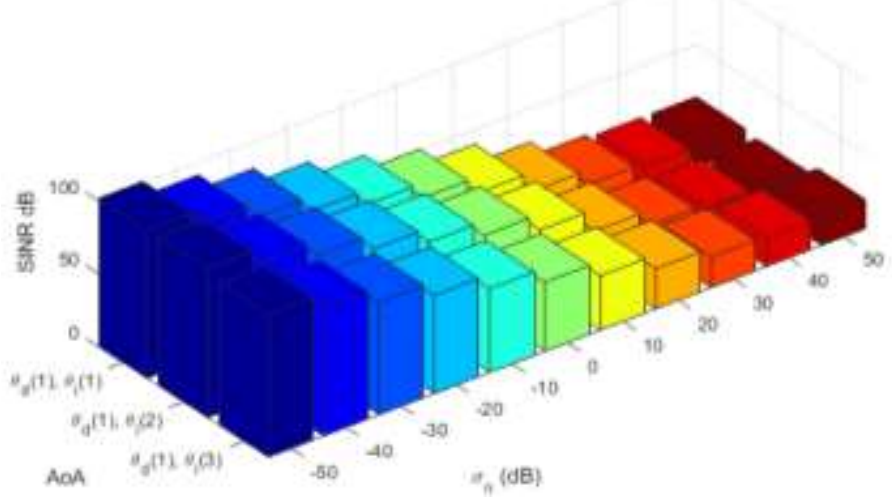

(d)

Fig. 2: Beampattern analysis of MVDR BF with $\mathrm{L}=8, \mathrm{~d}=\lambda / 2$ and SNOIs $=1,2,3$ 
TABLE III

MVDR PERFORMANCE ANALYSIS FOR L $=8, \mathrm{D}=\Lambda 2$ AND $\Sigma_{N}{ }^{2}=-50,0,50 \mathrm{DB}$.

\begin{tabular}{ccccccccccccccccc}
\hline \hline$\sigma_{n}{ }^{2}$ & $\theta_{s}$ & $\theta_{i}$ & Null & SINR & - & $\sigma_{n}{ }^{2}$ & $\theta_{s}$ & $\theta_{i}$ & Null & SINR & - & $\sigma_{n}{ }^{2}$ & $\theta_{s}$ & $\theta_{i}$ & Null & SINR \\
\hline-50 & & & -101 & 101.3 & - & -50 & & \multirow{2}{*}{-105} & 101.9 & - & -50 & & $-30^{\circ}$ & -110 & 97.1 \\
0 & $-20^{\circ}$ & $0^{\circ}$ & -51 & 51.0 & - & 0 & $0^{\circ}$ & $-15^{\circ}$ & -55 & 52.2 & - & 0 & $30^{\circ}$ & $0^{\circ}$ & -59 & 47.1 \\
50 & & & -14.4 & 14.4 & - & 50 & & & -38 & 12.6 & - & 50 & $50^{\circ}$ & -48 & 19.3 \\
\hline \hline
\end{tabular}

This scenario is done to show how MVDR beamformer deal with interference source in case of SNOIs incre-ases with $\sigma_{n}{ }^{2}$ on the performance of MVDR for a single desired user at $\theta_{s}$ and different number of interference signals $\left(\theta_{i}\right)$ with $\mathrm{L}=8$, $d=\lambda / 2$. Figure $2(d)$ compares the SINR of the MVDR beamformer for various amounts of ${\sigma_{n}}^{2}$ ranging from -50 to 50 $\mathrm{dB}$ with $\mathrm{SOI}=\{1\}$ and $\mathrm{SNOIs}=\{1,2,3\}$ for the fixed number of elements. One can observe from this plot is that the MVDR performance achieves the deepest negative power with higher SINR as signal power much higher than the noise, in case of signal power same label to the noise power still MVDR beamformer give higher SINR with maximum gain directed to the look angle. In this manner, MVDR beamformer found to have good performance even for the interference source increase with higher $\sigma_{n}{ }^{2}$. In short, reducing the effect of interference arriving outside the majorlobe. This interference reduction increases the capacity of the communication systems. Pattern nulling at specific directions suppresses the interference from other sources located at these directions. It can be clearly seen that the average SINR for $\sigma_{n}{ }^{2}=-50 \mathrm{~dB}$ is nearly above $100.1 \mathrm{~dB}$. Whereas, the SINR is heavily decreased as the $\sigma_{n}{ }^{2}$ is going up to reach $50 \mathrm{~dB}$ with average SINR around $15.4 \mathrm{~dB}$. The fundamental reason can be described as follows. If the value of $\sigma_{n}^{2}$ is low, the assessed statistics become more accurate with higher SINR and deepen null. Nevertheless, if the value of $\sigma_{n}{ }^{2}$ is high, MVDR cannot get a reliable estimation of the signal statistics, which affects the MVDR performance.

\section{CONCLUSION}

Many current wireless communication systems still depend on older technology. Recent efforts are being excited to modify wireless communication system to include adaptive antenna system. MVDR algorithm that has gained significance in the wireless cellular communication system due to its capability to suppress co-channel and neighboring channel interference with noise reduction and raised SINR helps to improve system capacity. In this work, the SINR and beampattern of the MVDR beamformer for varying noise labels and different angular separation between the SOI and SNOI have been investigated. The dependency of the MV-DR SINR on noise and interferences is also shown based on numerical simulations. The results obtained usin-g MVDR algorithm has the best beam formed pattern in suppressing the interference and noise reduction with accurate majorlobe to the look angle of the real user direction even with $\sigma_{n}{ }^{2}$ high. MVDR shows the capability of nulling unwanted signals with low power level, and perfect accuracy even in the case of mult-iple interferences. The MVDR performance, reduces as the noise variance label is increasing. The investig-ations into this area are still ongoing to extend the results of this work to enhance MVDR algorithm.

\section{ACKNOWLEDGMENT}

This work was supported by Universiti Malaysia Pahang, through the Fundamental Research Grant Scheme (FRGS) funded by Ministry of Education (RDU 140129).

\section{REFERENCES}

[1] Cisco Visual Networking Index, "Global mobile data traffic forecast update, 2013-2018," Cisco Systems Inc., San Jose, CA, USA, 2014.

[2] Ericsson Mobility Report, "On the Pulse of the Networked Society," Ericsson, Kista, Sweden, 2015.

[3] J. C. Liberti and T. S. Rappaport, Smart antennas for wireless communications: IS-95 and third generation CDMA applications: Prentice Hall PTR, 1999

[4] C. Garza. (2011) 4G Digest - 17.25 million BWA/WiMAX and 320 thousand LTE subscribers reached in Q1 2011 4G Digest. Available: http://www.maravedis-bwa.com/Issues/6.16/Readmore1.html

[5] M. A. Halim, Adaptive array measurements in communications, 1 st ed. Norwood, MA, USA: Artech House Publichers, 2001.

[6] J. Okkonen, "Uniform Linear Adaptive Antenna Array Beamforming Implementation with a Wireless Open-Access Research Platform," M.S. thesis, Dept. Computer Science And Engineering, Univ. of Oulu, May. 2013.

[7] C. Pan, J. Chen, and J. Benesty, "Performance study of the MVDR beamformer as a function of the source incidence angle," IEEE/ACM Trans. on Audio, Speech, and Lang. Process, vol. 22, pp. 67-79, 2014.

[8] A. O. Khaldoon, M. M. Rahman, R. B. Ahmad, and L. A. Hassnawi, "Enhanced uniform linear array performance using modified minimum variance distortionless response beamformer algorithm," presented at the 2nd International Conference on Electronic Design (ICED), 2014 http://dx.doi.org/10.1109/iced.2014.7015798

[9] J.-R. Lin, Q.-c. Peng, and H.-z. Shao, "On diagonal loading for robust adaptive beamforming based on worst-case performance optimization," ETRI journal, vol. 29, pp. 50-58, Feb. 2007.

[10] D. G. Manolakis, V. K. Ingle, and S. M. Kogon, Statistical and adaptive signal processing: spectral estimation, signal modeling, adaptive filtering, and array processing. Norwood, MA, USA: Artech House, Inc., 2005.

[11] K. J. Das and K. K. Sarma, "Adaptive Beamforming for Efficient Interference Suppression Using Minimum Variance Distortionless Response," in International Conference on Advancement in Engineering Studies \& Technology, 2012, pp. 82-86.

[12] R. L.-U. Choi, R. D. Murch, and K. Letaief, "MIMO CDMA antenna system for SINR enhancement," IEEE Transactions on Wireless Communications, vol. 2, pp. 240-249, 2003. http://dx.doi.org/10.1109/TWC.2003.808961

[13] S. Serbetli and A. Yener, "Transceiver optimization for multiuser MIMO systems," IEEE Trans. on Sig. Process, vol. 52, pp. 214-226, 2004.

[14] D. Kum, D. Kang, and S. Choi, "Novel SINR-based user selection for an MU-MIMO system with limited feedback," ETRI Journal, vol. 36, pp. 6268, 2014. http://dx.doi.org/10.4218/etrij.14.0113.0267

[15] A. P. Rao and N. Sarma, "Performance Analysis of Differential Evolution Algorithm based Beamforming for Smart Antenna Systems," International Journal of Wireless and Microwave Technologies (IJWMT), vol. 4, pp. 19, 2014. http://dx.doi.org/10.5815/ijwmt.2014.01.01

[16] B.-J. Ku, D.-S. Ahn, S.-P. Lee, A. Shishlov, A. Reutov, S. Ganin, et al., "Radiation pattern of multibeam array antenna with digital beamforming for stratospheric communication system: statistical simulation," ETRI journal, vol. 24, pp. 197-204, 2002.

[17] E. Habets, J. Benesty, I. Cohen, S. Gannot, and J. Dmochowski, "New insights into the MVDR beamformer in room acoustics," IEEE Trans. on Audio, Speech, and Lang. Process, vol. 18, pp. 158-170, 2010. 
http://dx.doi.org/10.1109/TASL.2009.2024731

[18] M.-S. Lee, "Wideband Capon beamforming for a planar phased radar array with antenna switching," ETRI journal, vol. 31, pp. 321-323, 2009.

[19] L. C. Godara, Smart antennas. Boca Raton: CRC press, 2004. http://dx.doi.org/10.1201/9780203496770

[20] M. Souden, J. Benesty, and S. Affes, "A study of the LCMV and MVDR noise reduction filters," IEEE Trans. on Sig. Process, vol. 58, pp. 49254935, 2010.

[21] L. C. Godara, "Application of antenna arrays to mobile communications. II. Beam-forming and direction-of-arrival considerations," Proceedings of the IEEE, vol. 85, pp. 1195-1245, 1997. http://dx.doi.org/10.1109/5.611108

[22] G. Renzhou, "Suppressing radio frequency interferences with adaptive beamformer based on weight iterative algorithm," in Conference on Wireless, Mobile and Sensor Networks, (CCWMSN07), IET, 2007, pp. 648-651. http://dx.doi.org/10.1049/cp:20070231

[23] S. Haykin, Adaptive Filter Theory, 4th ed.: Prentice Hall, 2013.

[24] J. Capon, "High-resolution frequency-wavenumber spectrum analysis," Proceedings of the IEEE, vol. 57, pp. 1408-1418, 1969. http://dx.doi.org/10.1109/PROC.1969.7278

[25] Malaysian Communications and Multimedia Commission. (2011) SKMM-MCMC Annual Report. Available: http://www.skmm.gov.my/skmmgovmy/media/General/pdf/ 